\title{
Microsurgical anatomical study of the frontotemporal-zygomatic arch approach to the superior petroclival region
}

\author{
JING-YI ZHOU，JUN-HUI LU，XIU-YU ZHEN，WEI WANG，JING-FANG XU and WEI-WEI HU \\ Department of Neurosurgery, The Second Affiliated Hospital of Zhejiang University School of Medicine, \\ Hangzhou, Zhejiang 310009, P.R. China
}

Received July 28, 2011; Accepted August 22, 2011

DOI: 10.3892/etm.2011.345

\begin{abstract}
The aim of this study was to explore the microsurgical anatomy of the superior petroclival region, and thus provide an anatomical basis for operative approaches. The frontotemporal-orbitozygomatic approach was performed on 20 sides of 10 adult cadaver heads. In comparison to the range of the exposure with the removal of the anterior clinoid process, posterior clinoid process and part of the tip of the petrous bone, we measured the neurovascular course and their relation to the superior petroclival region. We found that the trochlear nerve goes through the edge of the tentorial marginal branch, taking $5.42 \mathrm{~mm}$ (4.26-6.96) away from the ophthalmic nerve. Exposing the arteria basilaris, above the middle piece the length of exposure is $15.52 \mathrm{~mm}$ (14.22-16.70), resulting in the posterior cerebral artery and the front part of the midbrain being completely exposed. There is little exposure on the front part of the pons and midbrain with a length of $5.6 \mathrm{~mm}$ (4.38-6.82). Removing the partial petrosal bones, the inferior segment of the basal artery is exposed, while 4 other nerves cab also be observed: Cranial, abducens, facial and vestibulocochlear. The frontotemporal-zygomatic arch approach can clearly expose the superior petroclival region. Obtaining more information on the relationship between the location of these structures, is therefore helpful in improving the safety and success of surgery in this region.
\end{abstract}

\section{Introduction}

Advances in microsurgical instruments and refinement of surgical techniques have made it possible to operate on the frontotemporal-zygomatic arch (1). The range of this approach can reach the anterior cranial and middle cranial base regions, posterior cranial fossa and up to $1 / 3$ of the clival area (2). The petroclival region, a clinically significant and complicated

Correspondence to: Dr Wei-Wei Hu, Department of Neurosurgery, The Second Affiliated Hospital of Zhejiang University School of Medicine, Hangzhou, Zhejiang 310009, P.R. China

E-mail: zjy1972@126.com

Key words: frontotemporal-orbitozygomatic approach, superior petroclival region, microsurgical anatomy area, can be divided into 3 parts: The superior, located in the starting point of the cranial nerve stem brain, the middle, located between the starting point of the trigeminal and glossopharyngeal nerves, and the inferior, located under the starting point of the glossopharyngeal nerve also including the foramen occipitale magnum $(3,4)$. The postauricular, transpetrous and presigmoid approaches combine a supra/ infratentorial exposure with partial petrosectomy to access the third to twelfth cranial nerves and the anterolateral to the brainstem. It provides a shorter working distance to the large petrosal, petroclival, cerebellopontine and cerebellomedullary cisternal lesions (5). The microanatomy for surgery on the superior petroclival region through the frontotemporal-zygomatic arch approach remains unclear (6). Therefore, the aim of the present study was to provide a foundation of microanatomy for surgery on this region via the frontotemporal-zygomatic arch approach. We show that this approach provides excellent exposure of the superior petroclival region with minimal brain retraction and nerve injury.

\section{Materials and methods}

Specimens. Ten adult cadaver heads (20 sides) fixed by $10 \%$ formalin, 7 male (16 sides) and 3 female (6 sides), were used in this study. None of the cadaver heads used had any malformations, surgical traumas, disease or any surgical traces in the skull. All the specimens used were obtained from people who had donated their body to science and research. Ethical permission for this study was granted by the Ethical Committee of Zhejiang University School of Medicine (Hangzhou, China).

Surgical instruments. Surgical instruments used included an operating head-frame, high-speed drill, common craniocerebral surgical instruments, microsurgical instruments, an electric suction apparatus, and a pair of scissors and razor.

Observing and measuring equipment. These included an operating microscope, digital camera, triangle, sliding caliper (degree of accuracy $0.02 \mathrm{~mm}$ ), measure gauge, conimeter (degree of accuracy: 1), galvanizer etc.

Methods. The frontotemporal-zygomatic arch approach includes the following surgical steps: Firstly, it begins with normal surgery on the skull, exposing the bone flap and 
applying the orbitozygomatic osteotomy technique to remove the frontotemporal-zygomatic arch.

The first step to the exposure of the superior petroclival region, is extradural, the anterior processus clinoideus resection. It must be carefully executed in order to protect the crucial structures which surround it, while removing the processus clinoideus anterior. The structures include the optic nerve on the medial wall, the oculomotor nerve on the lateral wall and the internal carotid artery on the inferior wall. After removing the processus clinoideus anterior, the field of vision is greatly improved. Then gradually retract the frontal lobe, the optic chiasm and the anterior communicating artery complex, thus exposing the pituitary stalk behind the optic chiasm. Unfolding the lilliquist membrane between the internal carotid artery and optic nerve, along the posterior communicating and cerebral arteries to reach the P1-2 junction, eventually reveals the basilar artery bifurcation and the petroclival region.

By observing the course, distribution and relationship between the nerves and blood vessels, a number of significant data were measured. With the removal of the processus clinoideus anterior, processus dorsi sellae and partial petrosal bones, the range of exposure were measured.

\section{Results}

Exposure of optic nerve. Though the optic nerve is not located on the superior petroclival region, is still an essential nerve through the frontotemporal-zygomatic arch approach. During the surgery the distal end branch of the arteria cerebri aborales and the furcation of the internal carotid artery can be observed after the following steps: Unfolding the cerebral dura mater, retracting the frontal and temporal lobe, opening the lateral fissure of telencephalon by an arachniod knife, separating the sylvian vein, dragging the sylvian vein away to the side of temporal lobe, separating the lateral fissure and identifying the remote branch of the middle cerebral artery bifurcation of internal carotid artery by being separated. Fig. 1 shows the interspace between the optic nerve and the posterior communicating artery. Further steps in accordance with the method exposed the basilar artery before removing the processus clinoideus anterior, in which the length of the optic nerve was $10.22 \mathrm{~mm}(9.20-11.94)$, and after the removal of the processus clinoideus anterior the length was $22.62 \mathrm{~mm}(20.36-24.48)$.

Exposure of oculomotor and trochilear nerves. The oculomotor nerve went through the superior cerebellar and posterior cerebral arteries (Fig. 2). The distance of the inferior extremity between the posterior cerebral artery and the lower edge of the cerebellar tentorium was approximately $18.32 \mathrm{~mm}$ (17.04-19.88). The trochlear nerve went through the inferior part of the caudal colliculus located on the mesencephalicus lateralis dorsalis, reaching the inferior edge of the cerebellar tentorium at the border between the cerebral peduncle and the cerebellar tentorium. The distance was approximately $6.02 \mathrm{~mm}$ (4.28-6.98) after the trochlear nerve went into the free edge of the cerebellar tentorium.

Relative vessels on the superior petroclival region. The artery of the superior petroclival region includes the posterior cere-

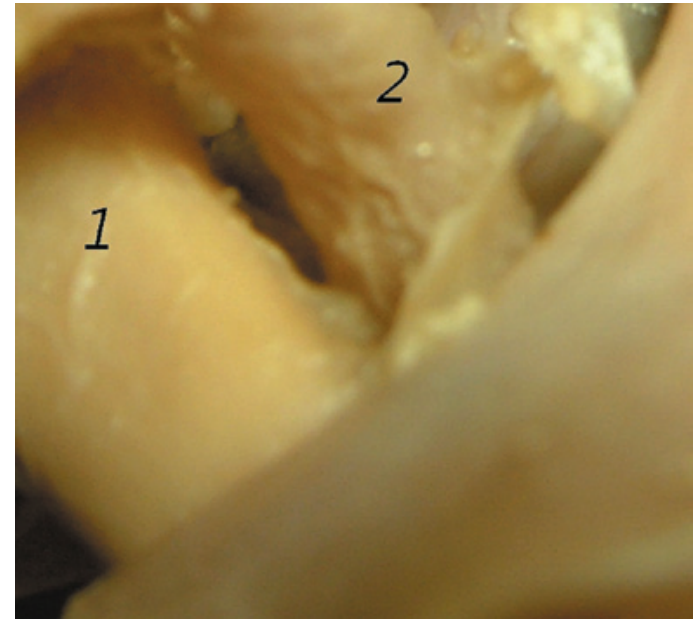

Figure 1. Internal carotid artery and optic nerve. 1, optic nerve; 2, internal carotid artery.

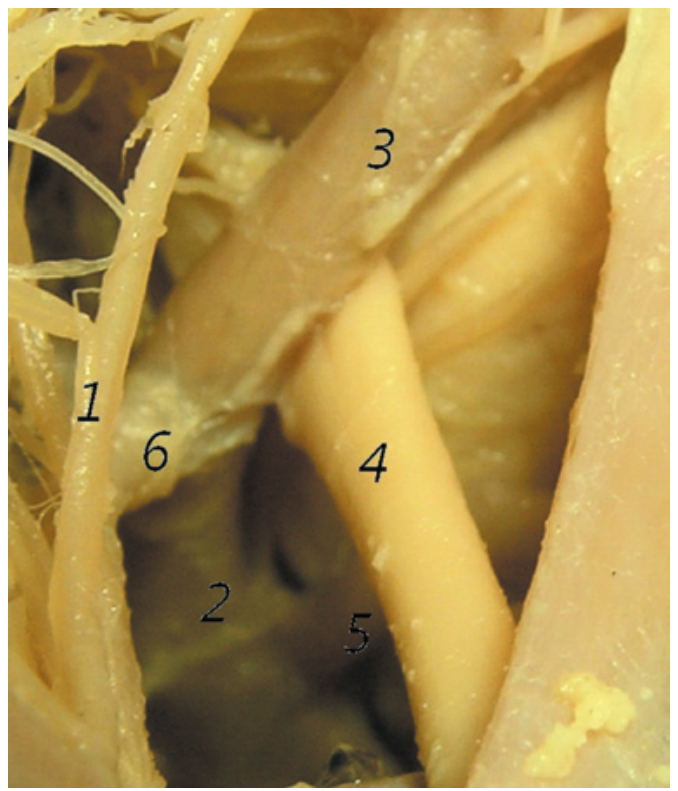

Figure 2. Exposure of superior petroclival region. 1, choroidal arteries; 2 , basal artery; 3 , posterior cerebral artery; 4, oculomotor nerve; 5 , superior cerebellar artery; 6 , posterior communicating artery.

bral, superior cerebellar and basilar arteries. According to its course, the posterior cerebral artery can be divided into the frontal, lateral and posterior segments. The frontal segment of the midbrain was completely exposed, with the length from the initial point of the basilar artery, and the junction of the posterior communicating and posterior cerebral arteries being $6.58 \mathrm{~mm}$ (5.42-7.84). The superior cerebellar artery can also be divided into 4 segments according to its course: The anterior pontomesencephalic below the oculomotor nerve, the lateral pontomesencephalic course, below the trochlear and above the trigeminal nerve, the cerebellomesencephalic course in the groove between the cerebellum and the upper brain stem, and the cortical which is distributed on the cerebellar surface. The length of exposure of the anterior pontomesencephalic segment in this approach of the superior cerebellar artery was approximately $5.6 \mathrm{~mm}$ (4.38-6.82). However, the basilar artery 
was well exposed, with a length of exposure above the middle segment of approximately $15.52 \mathrm{~mm}$ (14.22-16.70).

Results of relative structure removed. The approach was conducted in the superior petroclival region, going through the metasellar and alar bones. As the approach deepened into the brain and narrowed the surgical space, the anterior and posterior clinoid processes had to be removed. The resection of the anterior clinoid process, had no effect on the petroclival region the structure revealed, though it increased the light in the surgical area. However, the removal of the posterior clinoid process, inevitably results in the hemorrhage of the sinus cavernosus due to the oculomotor nerve going through its superolateral part. The removal of the posterior clinoid process can enhance the exposure of the basilar artery, though affecting less the exposure of the petroclival region. The removal of the apex of the petrous part, can enhance the exposure to the middle and inferior parts of petroclival region which has little influence on the superior petroclival region. Some structures, such as the trigeminal, vestibulocochlear, facial and abducent nerves can be observed.

\section{Discussion}

Although CSF cytology, CT and MRI scanning are the most essential techniques used for the diagnosis of brain tumors (7), surgery on the superior petroclival region has many difficulties. Neurosurgeons use anterior aspect approaches including transcavernous transpetrous, transcrusal and subtemporal keyhole $(4,8,9)$. Currently, the subtemporal approach is used more compared to the others (10). With these approaches, neurosurgeons have limited access and cannot achieve complete results following surgery for meningioma and trigeminal neuroma near the parasellar region and interpeduncular fossa (11). In addition, a frontotemporal craniotomy has been reported to provide a wide exposure of the anterior temporal base, thus allowing oblique access to the interpeduncular cistern with minimal brain retraction (12). This study provides an experimental basis for the temporal orbital-zygomatic arch approach to the petroclival region, thus fully exposing the petroclival region.

Previously, the subtemporal approach was uded through the cerebellar tentorium, easily inducing severe temporal lobe stretch (13). Studies have been carried out to describe the meningeal anatomy related to this approach as well as the dural incisions, to develop a subtemporal interdural approach to dumbbell-shaped trigeminal schwannomas that effectively converts a multiple-compartment tumor into a single-compartment tumor (14). By removing the petrous apex of the temporal bone and zygomatic arch, doctors could achieve greater exposure. This can significantly reduce the traction of the temporal lobe, and enlarge the surgical perspective as well as the exposure of the petroclival region (15). Hakuba et al were first to propose the orbital zygomatic arch infratemporal approach in 1986. Sixteen patients with parasellar tumors, 9 patients with basilar tip aneurysms, and 1 patient with a P-1 distal aneurysm, were operated on using this orbitozygomatic infratemporal approach, with excellent results (16). Deliberately excluding the microsurgical aspects of the lesions treated, doctors observed that the frontotemporal-orbitozygomatic approach was principally indicated for lesions requiring a multidirectional approach, such as sphenopetroclival tumors, aneurysms of the basilar tip and intracavemous lesions, while the frontotemporal-orbital approach proved to be excellent for more medial lesions, such as meningiomas of the luberculum sellae and cramopharyngiomas (17). Standard approaches to rostral middle fossa, such as the subtemporal, pterional, or orbitozygomatic, require significant brain retraction or manipulation of the temporalis muscle. Ong et al found that the endoscopic sublabial transmaxillary approach provides safe and direct access to this region (18). In comparison to subtemporal approach, the present study found that tumors in the superior petroclival region can grow to the posterolateral or middle petroclival regions. However, when the main body of the tumor was in the superior petroclival region, and grew to the direction of the saddle and cavernous sinus, the frontotemporal-zygomatic arch approach has more advantages. With the endoscope in the promotion of brain surgery, the surgery can have the same operating range as the frontotemporal-zygomatic arch approach, which can reach the posterior cavernous sinus, suprasellar and superior petroclival regions.

In summary, the endoscope is currently used more as an aid, as it still cannot replace the role of the microscope. Surgical limitations can be overcome by combining the surgical microscope and endoscope (19). The frontotemporalzygomatic arch approach to the petroclival region significantly exposes the various parts of the structure, and can be used to reach the anterior petroclival region. However, it should be noted that this leads to cerebrospinal fluid leakage and intracranial infection. The application of this approach requires further research.

\section{Acknowledgements}

This research study was supported by the Health Bureau of Zhejiang Province (no. 2007A089).

\section{References}

1. Schwartz MS, Anderson GJ, Horgan MA, Kellogg JX, McMenomey SO and Delashaw JB Jr: Quantification of increased exposure resulting from orbital rim and orbitozygomatic osteotomy via the frontotemporal transsylvian approach. J Neurosurg 91: 1020-1026, 1999.

2. Campero A, Campero AA, Socolovsky M, et al: The transzygomatic approach. J Clin Neurosci 17: 1428-1433, 2010.

3. Fournier HD, Mercier P and Roche PH: Surgical anatomy of the petrous apex and petroclival region. Adv Tech Stand Neurosurg 32: 91-146, 2007.

4. Wang H, Zhou F, Zhang R, Zhong P and Tan D: Opening cranial cisterns by the anterior subtemporal keyhole approach to the superior petroclival region: anatomical study and comparative analysis. Surg Neurol 72: 124-130, 2009.

5. Behari S, Tyagi I, Banerji D, et al: Postauricular, transpetrous, presigmoid approach for extensive skull base tumors in the petroclival region: the successes and the travails. Acta Neurochir (Wien) 152: 1633-1645, 2010

6. Jian FZ, Santoro A, Innocenzi G, Wang XW, Liu SS and Cantore G: Frontotemporal orbitozygomatic craniotomy to exposure the cavernous sinus and its surrounding regions. Microsurgical anatomy. J Neurosurg Sci 45: 19-28, 2001.

7. Liu J, Jia H, Yang Y, Dai W, Su X and Zhao G: Cerebrospinal fluid cytology and clinical analysis of 34 cases with leptomeningeal carcinomatosis. J Int Med Res 37: 1913-1920, 2009.

8. Roche PH, Mercier P and Fournier HD: [Temporopolar epidural transcavernous transpetrous approach. Technique and indications]. Neurochirurgie 53: 23-31, 2007. 
9. Horgan MA, Delashaw JB, Schwartz MS, Kellogg JX, Spektor S and McMenomey SO: Transcrusal approach to the petroclival region with hearing preservation. Technical note and illustrative cases. J Neurosurg 94: 660-666, 2001.

10. Pichierri A, D'Avella E, Ruggeri A, Tschabitscher M and Delfini R: Endoscopic assistance in the epidural subtemporal approach and Kawase approach: anatomic study. Neurosurgery 67: ons29-37, 2010.

11. Behari S, Jaiswal S, Garg P and Jaiswal AK: Bilateral eyebrow incision, mini-supraorbital craniotomy with extended frontobasal approach for extensive anterior and middle cranial fossa skull base tumors. Acta Neurochir (Wien) 153: 527-531, 2010.

12. Deda H and Ugur HC: Zygomatic anterior subtemporal approach for lesions in the interpeduncular cistern. Skull Base 11: 257-264, 2001.

13. Chernov MF: Far posterior subtemporal approach to the dorsolateral brainstem and tentorial ring: technique and clinical experience. Neurosurgery 54: 1028-1029, 2004.

14. Youssef S, Kim EY, Aziz KM, Hemida S, Keller JT and van Loveren HR: The subtemporal interdural approach to dumbbell-shaped trigeminal schwannomas: cadaveric prosection. Neurosurgery 59: ONS270-278, 2006.
15. Goel A: Extended lateral subtemporal approach for petroclival meningiomas: report of experience with 24 cases. Br J Neurosurg 13: 270-275, 1999.

16. Hakuba A, Liu S and Nishimura S: The orbitozygomatic infratemporal approach: a new surgical technique. Surg Neurol 26 271-276, 1986.

17. Santoro A, Salvati M, Vangelista T, Delfini R and Cantore GP: Fronto-temporo-orbito-zygomatic approach and variants. Surgical technique and indications. J Neurosurg Sci 47: 141-147, 2003.

18. Ong BC, Gore PA, Donnellan MB, Kertesz T and Teo C: Endoscopic sublabial transmaxillary approach to the rostral middle fossa. Neurosurgery 62: 30-37, 2008.

19. Inoue K, Seker A, Osawa S, Alencastro LF, Matsushima T and Rhoton AL Jr: Microsurgical and endoscopic anatomy of the supratentorial arachnoidal membranes and cisterns. Neurosurgery 65: 644-665, 2009. 